\title{
AN UNUSUAL SPURT OF SALMONELLA ENTERICA SEROVAR PARATYPHI B INFECTION DETECTED IN A TERTIARY CARE HOSPITAL IN KOLKATA
}

\author{
Indrani Bhattacharyya ${ }^{1}$, Soumya Dasgupta², Dilip Kumar Bera ${ }^{3}$
}

${ }^{1}$ Associate Professor, Department of Microbiology, Calcutta National Medical College, Kolkata, West Bengal, India.

${ }^{2}$ Special Duty Medical Officer, Department of Microbiology, Basirhat Superspeciality Hospital, Basirhat, West Bengal, India. 3 Professor and HOD, Department of Microbiology, School of Tropical Medicine, Kolkata, West Bengal, India.

\begin{abstract}
BACKGROUND
Salmonella enterica serovar Paratyphi B is a member of the genus Salmonella of the Enterobacteriaceae family. They cause enteric fever as well as gastroenteritis in humans. Salmonella enterica serovar Typhi and Salmonella enterica serovar Paratyphi A are the predominant types of Salmonella responsible for enteric fever in India. However, this study is based on an unusual spurt of Salmonella enterica serovar Paratyphi B infection, which had not been isolated in our laboratory for last four years.
\end{abstract}

\section{MATERIALS AND METHODS}

Blood cultures were taken from the patients. After incubation, routine subculture was done on MacConkey agar and blood agar media. Relevant biochemical tests were done for identification of the growth followed by agglutination for serotyping. Finally, antibiotic sensitivity tests were done.

\section{RESULTS}

The causative organism was diagnosed as Salmonella enterica serovar Paratyphi B by blood culture, biochemical tests and serotyping. No major drug resistance was detected.

\section{CONCLUSION}

Salmonella enterica serovar Paratyphi B is an uncommon cause of enteric fever. We must keep our eyes and ears open every time we isolate non-lactose fermenting gram-negative bacilli from blood or stool. Unless this awareness is maintained, these infections will be very difficult to contain and will become a serious problem in the near future.

\section{KEY WORDS}

Enteric Fever, Paratyphi B, Enterobacteriaceae, Blood Culture, Gram-Negative Bacilli.

HOW TO CITE THIS ARTICLE: Bhattacharyya I, Dasgupta S, Bera DK. An unusual spurt of salmonella enterica serovar paratyphi B infection detected in a tertiary care hospital in Kolkata. J. Evolution Med. Dent. Sci. 2018;7(29):3329-3331, D0I: $10.14260 /$ jemds/2018/750

\section{BACKGROUND}

Normally, during the summer and monsoon months, the bacteriology laboratory in the tertiary care hospital in Kolkata encounters one or two cases of enteric fever. Majority of the cases are caused by Salmonella typhi, which is usually confirmed by agglutination with appropriate antisera or by the Vitek system. However, during last summer, we were faced with a unique situation. Four different blood culture samples were tested and all four turned out to be Salmonella enterica serovar Paratyphi B. Surprisingly, all these cases were severe enough to merit hospitalisation. This study aims to analyse this unusual spurt of Paratyphi B infection, which was not only rare but severe also. It may also be predicted that under cover of these four cases, many more less severe cases were there which did not require hospitalisation and some cases which may have attended other hospitals.

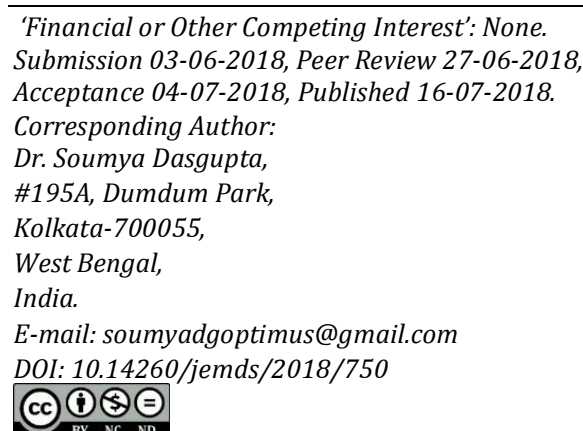

Salmonella enterica serovar Paratyphi B belongs to the family Enterobacteriaceae and genus Salmonella. It causes paratyphoid fever and gastroenteritis and can be isolated from animal hosts. A few large food-borne outbreaks have been reported from United Kingdom in 1988 (Anonymous 1994) ${ }^{1}$ and France in 1993 (Desenclos et al, 1996). ${ }^{2}$

The infection in all cases was either food-borne or waterborne. Unlike Shigella where only 100 organisms can produce infection, about 200 to $10^{6}$ colony forming units are required for producing infection in case of Salmonella. ${ }^{3}$ Neutralisation of acidity in the stomach, alteration of bowel flora, loss of integrity of the bowel mucosa etc. due to any reason facilitates Salmonella infection.

Salmonella typhi, paratyphi A and paratyphi B have no animal reservoir. Apart from classical feco-oral transmission, sexual transmission among homosexual males has been reported. ${ }^{4}$ Health care workers may get infected from the patients or during processing of specimens in the laboratory. Luckily, the status of hygiene has improved markedly in the developing countries worldwide. However, some endemic pockets do exist, and surprisingly enough new cases appear occasionally in previously non-endemic locales.

This study is based on one such spurt of Salmonella enterica serovar Paratyphi B infection, which had not been isolated in our laboratory for last four years. The last case was detected four years ago- a single case from a patient admitted with prolonged fever, anorexia and weakness. 


\section{MATERIALS AND METHODS \\ Study Period}

This is a descriptive study. The first sample was isolated in mid-May 2016, and the fourth sample was obtained in the end of June 2016. After this, for the next one year no other isolates of Salmonella paratyphi B were obtained.

\section{Material}

Conventional blood culture samples from patients admitted in a tertiary care hospital in Kolkata.

\section{Method}

Blood culture bottles were incubated at $37^{\circ} \mathrm{C}$ and routinely subcultured after 24 hours in MacConkey agar and blood agar media. Gram stain and hanging drop preparations were done from the growth obtained. Relevant biochemical tests were done for identification of the growth followed by agglutination for serotyping. Finally, antibiotic sensitivity tests were done.

\section{RESULTS}

After incubation of the plates at $37^{\circ} \mathrm{C}$ for 24 hours, growth was obtained. MacConkey agar showed effuse, non-lactose fermenting colonies. Blood agar showed convex, moist, greyish colonies with entire edges. The colonies were translucent and non-haemolytic.

Routine Gram stain showed Gram-negative bacilli, nonsporing and haphazardly arranged.

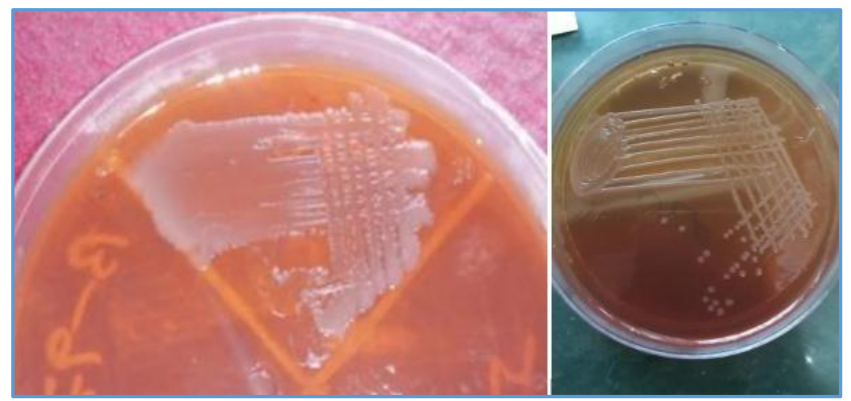

Figure 1. Growth on MacConkey Agar

Hanging drop preparation showed motile organism. No special motility pattern was noted.

\section{Biochemical Tests}

Indole -Neg; TSI-K/A with $\mathrm{H}_{2} \mathrm{~S}$ production along the stab line only; Urease -Neg, Citrate +ve, Oxidase -Neg, Lysine +ve, Ornithine +ve, Arginine -Neg, PPA -Neg, Reduced nitrate to nitrite, ONPG -ve, Glucose -Acid+ gas; Sucrose -ve; Mannitol, Arabinose fermented; Adonitol -ve.

Vitek 2 also showed that the organisms belonged to Salmonella species.

Agglutination reaction: Serotyping was done using specific antisera. The organisms were positive for Poly 0 (Salmonella genus). They failed to agglutinate Group 2 (O Ag group B) and Group 9 (O Ag group D1) antisera, but agglutinated serogroup 4 antisera (O Ag group B). ${ }^{5}$ So, the organisms belonged to Salmonella serogroup 04, of which paratyphi $\mathrm{B}$ is the most common human pathogen causing enteric fever.

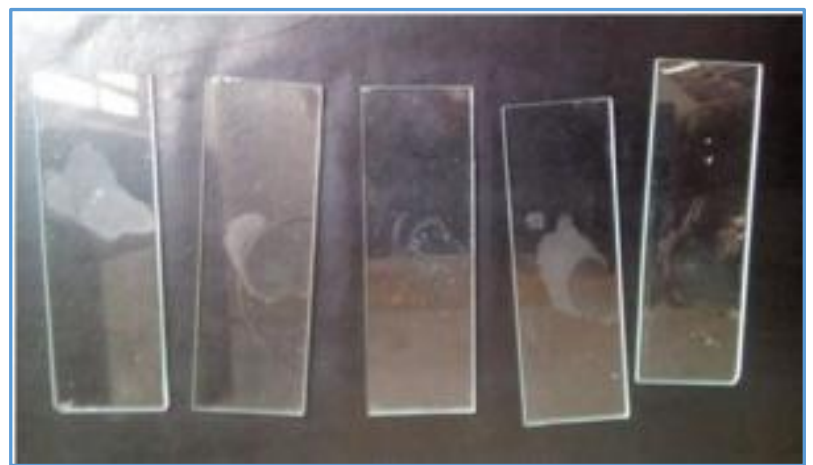

Figure 2. From Left-to-Right: Salmonella Poly 0 antiserumagglutination, Salmonella group 2 antiserum- no agglutination, Salmonella group 4 antiserumagglutination, Salmonella group 9 antiserum- no agglutination, Control with Normal Saline

\section{Antibiotic Sensitivity Test}

Performed by Kirby-Bauer method on Mueller-Hinton agar media. The following pattern was observed.

\begin{tabular}{|c|c|c|c|c|c|c|c|}
\hline 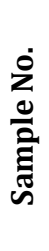 & 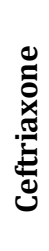 & 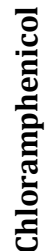 & 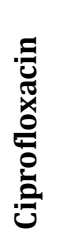 & & 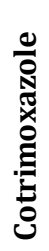 & 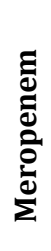 & 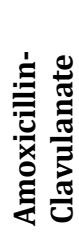 \\
\hline 1 & $S$ & $S$ & $S$ & $S$ & $S$ & $S$ & $S$ \\
\hline 2 & $\mathrm{~S}$ & $\mathrm{~S}$ & $\mathrm{R}$ & $\mathrm{S}$ & $\mathrm{R}$ & $\mathrm{S}$ & $\mathrm{R}$ \\
\hline 3 & $S$ & $S$ & $\mathrm{~S}$ & $S$ & $\mathrm{R}$ & $\mathrm{S}$ & $\mathrm{R}$ \\
\hline 4 & $S$ & $\mathrm{~S}$ & $S$ & $S$ & $\mathrm{R}$ & $\mathrm{S}$ & $\mathrm{R}$ \\
\hline
\end{tabular}

Neither similarity pattern nor any multi-drug resistance was observed.

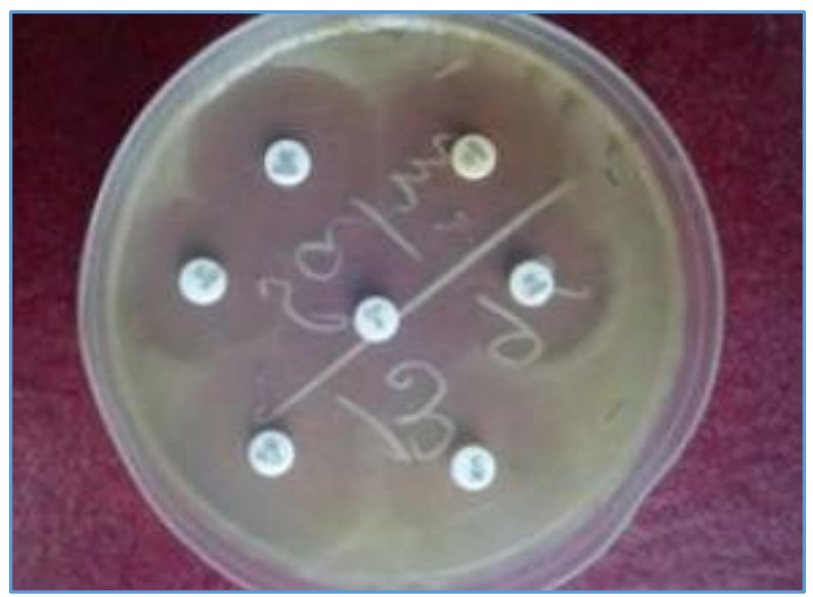

Figure 3. Drug susceptibility test on Mueller-Hinton Agar with Disk Diffusion Method

\section{DISCUSSION}

Paratyphi B is also known as schottmuelleri, but this synonym is rarely used now. The phage typing method of Anderson (1964) differentiates 55 phage types and the biotyping scheme of Duguid et al (1975) 56 full biotypes (Barker et al, 1988). 


\section{Two Groups of Strains Exist}

1. Some cause enteric fever in humans, 90 percent are dtartrate non-fermenting (dT-).

2. Others cause gastroenteritis in humans, usually $d$ tartrate fermenting $(\mathrm{dT}+$ ) (referred to as Salmonella Paratyphi B variant $\mathrm{L}(+)$-tartrate + or Salmonella Paratyphi B variant Java). ${ }^{6}$ Discrimination between strains of dT- (Paratyphoid-like) and dT+ (biotype Java, non-paratyphoid- like) strains of S. paratyphi B can be facilitated by MLEE, PFGE, IS200 fingerprinting and more recently by the presence of the sopE, sopD and sopE1 virulence genes (Prager et al, 2003). ${ }^{7}$ Lately, there is a spurt of biotype java infection and strains are mostly drug resistant.

\section{CONCLUSION}

The most important point that came to mind regarding this unusual spurt of Salmonella paratyphi B in blood was 'what was the source of infection?'. Patients were not from any one particular locality and were not related in any way. However, all four cases were from villages and the patients were used to open air defecation. Poverty was definitely another factor. There was lack of health education. They were all having a hand-to-mouth existence and the concept of cleanliness, hand washing and basic hygiene had no place in their lives. They had no idea about proper waste disposal, clean water and food hygiene. Thus, diagnosing cases and treating them successfully was not serving to solve the problem. What was needed was to attack the root cause. Widespread health education, simple cleanliness, hygienic disposal of wastes should be taught and practiced regularly in order to control and prevent such infections. ${ }^{8}$
Hence, medical personnel and health workers need to work hard in order to abolish these diseases and to ensure that such infections do not creep back into society and catch us unaware.

\section{REFERENCES}

[1] Salmonella in animal and poultry production. New Haw: Central Veterinary Laboratory 1992:1-40.

[2] Desenclos JC, Bouvet P, Benz-Lemoine E, et al. Large outbreak of Salmonella enterica serotype paratyphi B infection caused by a goats' milk cheese, France, 1993: a case finding and epidemiological study. Br Med J 1996;312(7023):91-4.

[3] David PA, Miller SI. Harrison's Principles of internal medicine. 19th edn. McGraw-Hill Education 2015: p. 1049.

[4] Reller ME, Olsen SJ, Kressel AB, et al. Sexual transmission of typhoid fever: a multistate outbreak among men who have sex with men. Clin Inf Dis 2003;37(1):141-4

[5] Old DC. Mackie \& McCartney Practical medical microbiology. 14th edn. 2012: p. 390.

[6] John TE. Salmonella. Topley and Wilson's Microbiology and microbial pathology. $10^{\text {th }}$ edn. 2006.

[7] Prager R, Rabsch W, Streckel W, et al. Molecular properties of Salmonella enterica serotype Paratyphi $\mathrm{B}$ distinguish between its systemic and its enteric pathovars. Journal of Clinical Microbiology 2003;41(9):4270-8.

[8] Park K. Preventive and Social Medicine. 23rd edn. Bhanot: 2015: p. 236-7. 